\title{
Signaling Properties of CD38 in the Mouse Immune System: Enzyme-dependent and -independent Roles in Immunity
}

\author{
Frances E Lund ${ }^{1}$ \\ ${ }^{1}$ Trudeau Institute, 154 Algonquin Ave., Saranac Lake, NY 12983 USA
}

\begin{abstract}
The 5th international CD38 meeting, held in Torino, Italy, spanned a range of topics from the role of CD38 as a signaling receptor in lymphocytic tumors to the importance of CD38-derived metabolites in $\mathrm{NAD}^{+}$metabolism, calcium signaling, and immune function. This meeting was particularly exciting as data were presented demonstrating that collaborative experiments between enzymologists, biochemists, cell biologists, immunologists, and clinicians have started to unravel the secrets of CD38 biology. It is now clear that all of the products of the CD38 enzyme reaction regulate calcium signal transduction in cell types as diverse as sea urchin oocytes and mammalian lymphocytes. It is also apparent that CD38 plays important immunomodulatory role(s), however there is still much debate on how CD38 mediates its immunoregulatory functions and whether the enzymatic products generated by CD38 are important for immunity. The data presented at this meeting have begun to resolve some of these controversies. First, CD38 regulates the function of leukocytes by enzyme-dependent and enzyme-independent mechanisms. Second, CD38 regulates inflammatory responses by modulating the activity of the responding leukocytes and by altering the activity of non-hematopoietic cells in the inflamed tissue. Finally, crosstalk between CD38 and other NAD ${ }^{+}$utilizing enzymes such as ART2, SIRT1, and PARP-1 impacts NAD+ homeostasis, inflammation, and immunity. Thus, immunity is regulated by CD38 in multiple and unexpected ways and the new research challenge will be to determine whether we can exploit the complex biology of CD38 to therapeutically regulate the immune system.
\end{abstract}

Online address: http://www.molmed.org

doi: 10.2119/2006-00099.Lund

\section{INTRODUCTION}

CD38 is a member of a family of enzymes that are highly conserved at the structural level and have been found in organisms as diverse as sea slugs, sea urchins, schistosomes, birds, and mammals $(1,2)$. The enzymes in this family are distinguished from all other known nucleotide-metabolizing enzymes by their ability to produce cyclic adenosine diphosphoribose (cADPR) from nicotinamide adenine dinucleotide $\left(\mathrm{NAD}^{+}\right)$. Cyclic ADP-ribose, 1st described more than 15 years ago by Lee et al. (3), induces calcium mobilization from ryanodine receptor-gated calcium stores in the endoplasmic reticulum (4). With the advent of cADPR antagonists $(5,6)$, many groups demonstrated that cADPR regulates numerous calcium signaling pathways in plants, invertebrates, and vertebrates $(1,7)$. Thus, it has become increasingly clear that one of the major functions of the CD38 family members is to produce cADPR. However, over the last decade, it also has become apparent that CD38 catalyzes the production of multiple nucleotide-based metabolites from $\operatorname{NAD}(\mathrm{P})^{+}$, including nicotinic acid adenine dinucleotide phosphate $\left(\mathrm{NAADP}^{+}\right)(8)$ and adenosine diphosphoribose (ADPR) (9), and that both of these metabolites play important roles in calcium signaling (1). Furthermore there is strong evidence that CD38 also can function as a plasma membrane signaling receptor in leukocytes (10).

A major focus of the 5th international CD38 meeting "The CD38 Ectoenzyme

Address correspondence and reprint requests to Frances $E$. Lund, Trudeau Institute, 154 Algonquin Ave., Saranac Lake, NY 12983. Phone: 518-891-3080; fax: 518-891-5126; e-mail: flund@trudeauinstitute.org.

Submitted November 10, 2006; accepted for publication December 7, 2006.

Family: Advances in Basic Science and Clinical Practice," held in Torino, Italy in June, 2006, was to elucidate how CD38 regulates immune responses to conventional antigens and autoantigens. Many of the laboratories studying human CD38 have focused on the receptor properties of this ecto-enzyme and these studies are reviewed in detail in this issue by Malavasi and his colleagues. In addition, a number of laboratories have used the mouse model to study the receptor and enzymedependent functions of CD38 in the immune system. This review will specifically highlight some of these newer data, focusing on the evidence from the mouse models indicating multiple roles for CD38 in regulating immune responses.

\section{RESULTS}

\section{CD38 Crosslinking Modulates B Cell Responses in an Enzyme-independent Fashion}

Leopoldo Santos-Argumedo first identified CD38 as a signaling receptor on 
mouse B lymphocytes more than a decade ago and was the first to show that antibodies to mouse CD38 induced the activation of mature mouse B lymphocytes $(11,12)$. His laboratory has continued to focus on the role of CD38 as a signaling receptor in mouse B lymphocytes and at the CD38 meeting SantosArgumedo presented unpublished data showing that crosslinking of CD38 not only induces the proliferation of mature follicular B cells but also enhances the proliferation and differentiation of the immature transitional 2 (T2) B cells. The effect of CD38 crosslinking on the differentiation of T2 cells appeared similar, although not identical, to the effects observed upon crosslinking the B cell antigen receptor $(\mathrm{BCR})$, suggesting that CD38 and the BCR use interrelated signal transduction machinery. Indeed, previous work from both Santos-Argumedo and myself showed that biologic effects observed upon crosslinking CD38 on immature and mature mouse B cells are dependent on expression of a functional BCR (13). However, as Santos-Argumedo and colleagues recently demonstrated, the signals induced in mature B cells upon CD38 or BCR crosslinking are not identical as PLC $\gamma 2$ inhibitors blocked signaling through the BCR but not through CD38 (14). Thus, these data suggest that while signaling through CD38 is dependent on the presence of a functional BCR complex, crosslinking of CD38 only triggers a subset of the signaling pathways initiated upon BCR crosslinking.

In addition to determining how CD38 crosslinking leads to the differentiation of T2 B cells, the Santos-Argumedo lab is also currently examining the effect of CD38 crosslinking on Toll-Like Receptor (TLR) signaling in mature B cells. Mouse $B$ cells express a number of different TLRs including TLR3, TLR4, TLR7, and TLR9 and stimulation of mature B cells with TLR ligands induces B cell activation, proliferation and differentiation into antibody secreting cells (15-18). Likewise, crosslinking of mature mouse $B$ cells with anti-CD38 in the presence of cytokines such as IL-4 induces prolif- eration (11) while activation of B cells with anti-CD38 in the presence of IL-5 induces the differentiation of the B cells into IgG1-secreting plasma cells (19). Santos-Argumedo showed several years ago that co-stimulation of B cells with anti-CD38 and the TLR4 ligand, LPS, induces an enhanced proliferative response (20), however the effect of coactivating B cells with TLR ligands and anti-CD38 on B cell differentiation was not examined. At the recent CD38 meeting, Santos-Argumedo presented data now submitted for publication showing that co-activation of B cells with LPS and anti-CD38 did not enhance the differentiation of B cells to plasma cells. In fact, a smaller proportion of B cells costimulated with anti-CD38 and TLR ligands differentiated into CD138 ${ }^{+}$(Syndecan) plasma cells compared with the $B$ cells that were stimulated with TLR ligands alone. In addition, the number of antibody-secreting cells and the amount of secreted IgM made by these cells was reduced in the B cells that were stimulated with TLR ligands plus anti-CD38 antibodies compared with the B cells activated with TLR ligands alone. Thus, the signals induced upon crosslinking of CD38 on mature mouse B cells appears to favor proliferation at the expense of differentiation.

Interestingly, the inhibitory effect of anti-CD38 stimulation on TLR-induced B cell differentiation did not appear to be dependent on the generation of CADPR by CD38, as B cell differentiation was still inhibited in LPS and anti-CD38 stimulated B cells that were treated with the cADPR antagonist, 8Br-cADPR. These preliminary data suggested that the receptor-mediated functions of CD38 can be separated from the enzyme activity of CD38. However, as described in the introduction, CD38 makes multiple calcium-mobilizing second messengers that could potentially influence signal transduction in B cells. To address this issue, my laboratory, in collaboration with the Schuber, Oppenheimer, and Santos-Argumedo groups, directly tested whether the receptor-mediated functions of murine CD38 are dependent on CD38 enzyme activity in B cells. We transfected the murine pro-B cell line $\mathrm{Ba} / \mathrm{F} 3$ with mouse CD38 and showed that CD38 crosslinking induced caspasedependent apoptosis of the CD38 transfected cells (21). However, treatment of the transfectants with a CADPR antagonist, 8Br-cADPR, with a CD38 inhibitor, AraF-NAD ${ }^{+}(22,23)$, or with the calciumchelating agents (BAPTA-AM and EGTA) did not block anti-CD38 induced apoptosis. Likewise, BAF/3 cells expressing a catalytically inactive form of CD38 (CD38 E229Q) were completely sensitive to the apoptotic signal induced upon CD38 crosslinking (21). Together, these data directly demonstrated that the formation of calcium-mobilizing metabolites by CD38 is not required for its receptor-mediated functions. Instead, our data suggested that the receptor-mediated properties of CD38 are dependent on tyrosine kinase activation and the localization of CD38 to the lipid raft microdomains on the plasma membrane (21). These results are in good agreement with data from the Sancho and Zubiar labs showing that CD38 signaling in human $\mathrm{T}$ cells occurs in the plasma membrane lipid raft microdomains (24-26). Importantly, however, the results also strongly suggest that CD38 has evolved to perform at least two independent functions.

\section{CD38 Regulates Leukocyte Trafficking by Producing CADPR}

Although CD38 crosslinking induces a number of biologic effects on mouse $B$ lymphocytes in vitro, there is still no evidence that B cells isolated from CD38 deficient $\left(\mathrm{Cd} 38^{--}\right)$mice make defective responses to BCR stimulation (27). Thus, it has been difficult to determine whether the receptor-mediated activities of CD38 are obligate for the function of mouse B lymphocytes. However, recent work from our laboratory now indicates that CD38 does regulate the in vivo functions of other hematopoietic cells, including dendritic cells (DCs), monocytes, and neutrophils $(28,29)$. In fact, we showed that 
trafficking of neutrophils to sites of infection and inflammation was dependent on CD38 expression on the neutrophils and that the in vitro chemotaxis of neutrophils to an array of inflammatory chemokines and chemoattractants was dependent on CD38 $(28,30)$. We observed similar results when we examined the in vivo and in vitro migration of $\mathrm{Cd} 38^{-/}$ monocytes and DCs (31). Interestingly, we also showed that CD38 regulates the calcium response of chemokine-stimulated neutrophils and DCs and that treatment of normal DCs or neutrophils with the cADPR antagonist, 8Br-cADPR, also blocked the calcium response and chemotactic potential of the cells $(30,31)$. Finally, we found that $8 \mathrm{Br}-\mathrm{cADPR}$ treatment blocked the migration of human peripheral blood neutrophils and monocytes to multiple chemoattractants (32). Together, these data strongly suggested that CD38 regulates cell trafficking by producing cADPR which controls chemokine receptor-mediated calcium signaling. In support of this model, we found that treatment of both mouse and human leukocytes with the CD38 substrate ana$\log 8 \mathrm{Br}-\mathrm{NAD}^{+}$blocked the in vitro chemotaxis of human and mouse leukocytes (30-32). Thus, we concluded that CD38, through its ability to catalyze an ADP-ribosyl cyclase reaction, likely plays an important role in inflammatory responses.

\section{CD38 Regulates Cell Trafficking by Activating Calcium Influx Through an ADPR-gated Cation Channel}

One of the observations made during our initial studies was that calcium influx in chemokine-stimulated neutrophils and DCs was dramatically reduced in leukocytes that lacked CD38 or had been treated with the CADPR antagonist $(30,31)$. This observation was initially surprising to us as cADPR was first identified as an activator of calcium release from intracellular calcium stores $(3,33)$. Indeed, extensive work from the laboratories of Hon-Cheung Lee and Tim Walseth showed that cADPR induced intracellular calcium release from $\mathrm{IP}_{3}$ receptor- independent stores in sea urchin egg extracts and subsequent work from a number of groups demonstrated that cADPR activated calcium release from Ryanodine Receptor-gated stores in the endoplasmic reticulum (4). However, there was a very nice study from the Guse laboratory (34), indicating that CADPR also controlled extracellular calcium influx through an unknown plasma membrane calcium channel. Excitingly, new studies from the group of Fleig and Penner have helped us to understand how cADPR activates calcium entry through the plasma membrane. Approximately five years ago, several laboratories simultaneously demonstrated that the nonselective cation channel, TRPM2, is activated upon binding of ADPR to the cytoplasmic NUDT9 domain found in TRPM2 (35-37). These groups found that relatively large quantities (high micromolar concentrations) of ADPR were needed to activate TRPM2 in whole cell patch clamp experiments, thus it was unclear to many investigators whether TRPM2 was activated under normal physiologic conditions by ADPR. However, the recently published experiments from Fleig and Penner's group showed that the amount of ADPR required to gate TRPM2 could be reduced by more than 100 -fold when cADPR was also present in the patch clamp pipette (38). The presence of cADPR on its own in the pipette did not appear to efficiently activate TRPM2, rather it appeared that CADPR acts to potentiate ADPR-gating of TRPM2 (38). This could be through direct binding of CADPR to TRPM2, however the structure of NUDT9 domain makes this appear unlikely (39), and, to date, there is no published evidence demonstrating that cADPR binds to other sites on the channel. Alternatively, experiments from $\mathrm{McHugh}$ et al. and Perraud and colleagues showed that the presence of cytosolic free calcium potentiates activation of TRPM2 by ADPR $(36,40)$. Thus, it is possible that CADPR facilitates ADPRmediated activation of TRPM2 by increasing cytosolic calcium levels. Importantly, similar results have been reported using other cell types $(41,42)$, thus it seems likely that a major function of CADPR may be to co-activate calcium influx through the ADPR-gated cation channel TRPM2.

Because cADPR appears to act as a "co-activator" of TRPM2 in transfected cell lines, we wanted to determine whether CADPR regulates ADPR-gated calcium influx in chemokine-stimulated primary leukocytes. Tim Walseth's laboratory synthesized a novel ADPR analog which was then tested by Andreas Guse's laboratory for its ability to block ADPRgated calcium entry in TRPM2-expressing cells. Excitingly, the ADPR analog efficiently blocked ADPR-dependent cation entry without affecting calcium entry through store-operated calcium channels. In experiments now submitted for publication, we tested the effect of this ADPR analog on calcium influx in chemokinestimulated leukocytes and found that the analog specifically blocked extracellular calcium influx and very efficiently inhibited the chemotaxis of both mouse and human leukocytes to a number of different chemoattractants. Together, these data strongly suggested that, in chemokinestimulated primary leukocytes, ADPR and CADPR together activate calcium influx through TRPM2. Furthermore the data suggest that CD38 regulates leukocyte trafficking by activating TRPM2.

\section{Inflammatory Responses and Airway Hyperreactivity Are Attenuated in Allergen-challenged $\mathrm{Cd} 38^{-/-}$Mice}

Given the important role that CD38 plays in leukocyte trafficking, we were not too surprised to find that some immune responses are attenuated in $\mathrm{Cd} 38^{-/-}$mice. In fact, the very earliest experiments using $\mathrm{Cd} 38^{-/}$mice demonstrated that $\mathrm{T}$ celldependent humoral immune responses were attenuated in $\mathrm{Cd} 38^{-/}$mice that were immunized with small quantities of protein antigens $(31,43)$. We subsequently showed that $\mathrm{Cd} 38^{-/}$DCs do not efficiently prime CD4 T cells, in large part because the $\mathrm{Cd} 38^{--}$DCs do not efficiently migrate from sites of inflammation to the draining lymph node where they would normally 


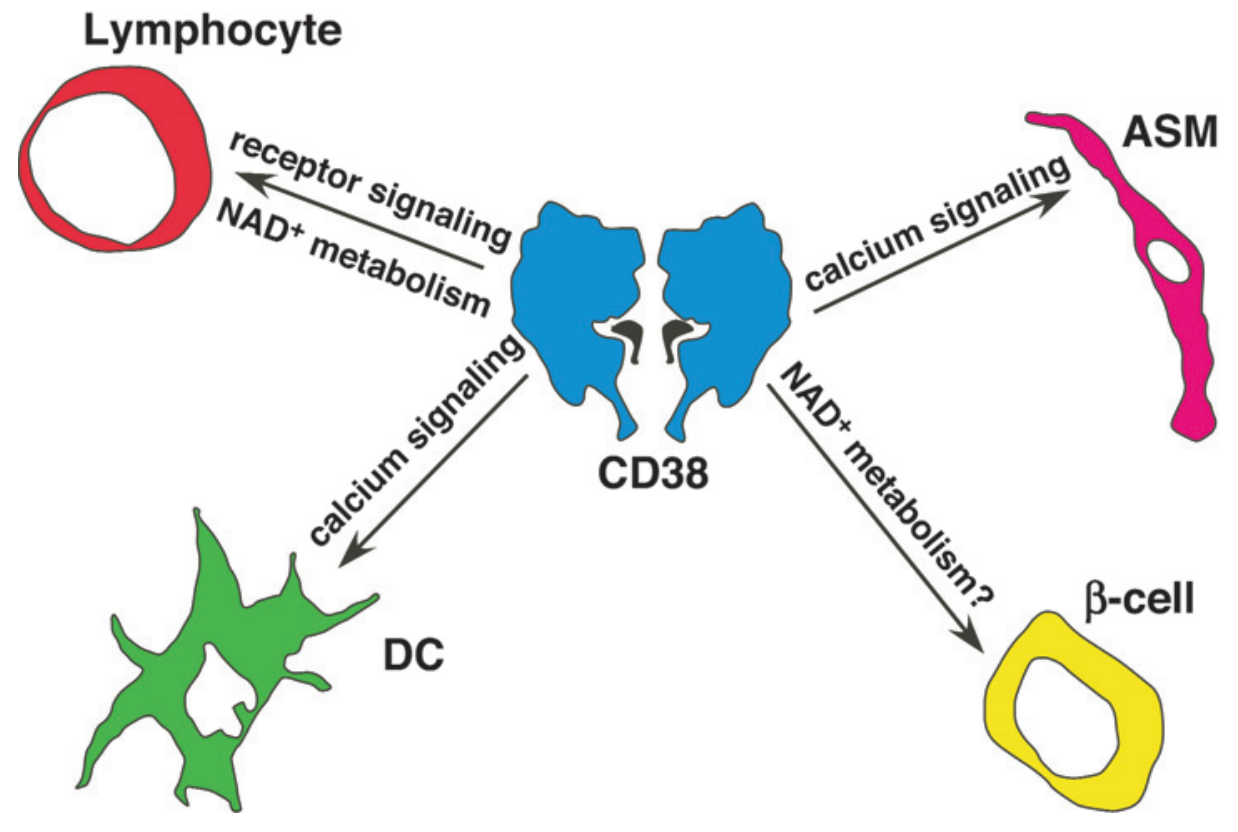

Figure 1. Multi-faceted roles for CD38 in immune responses and inflammation. Although CD38 was first described as a lymphocyte-restricted plasma membrane signaling receptor, it is now apparent that CD38 plays a diverse set of roles not only on lymphocytes but also on dendritic cells (DCs), granulocytes and multiple non-bone marrow derived cells, including airway smooth muscle cells (ASM) and pancreatic $\beta$-cells. In some cells types like ASM cells and DCs, CD38 regulates signaling by catalyzing the production of the calcium-mobilizing second messengers CADPR, NAADP ${ }^{+}$and ADPR. However, in other cells types, like $\beta$-cells and T cells, CD38 may indirectly regulate signaling by catabolizing $\mathrm{NAD}^{+}$and altering or preventing the activity of $\mathrm{NAD}^{+}$-metabolizing enzymes such as ART2, SIRT1, and PARP. Finally, in some cell types, including B lymphocytes, CD38 appears to regulate cell signaling by enzyme-independent mechanisms. Thus, while it is clear that CD38 modulates immune responses, the process is complex and involves multiple CD38expressing cell types and several different calcium-dependent and calcium-independent signaling pathways. Our new challenge will be to understand how all of these individual CD38-dependent signaling pathways interact with one another to regulate the global immune response to pathogens, allergens and autoantigens.

encounter naïve T cells (31). Recently, my laboratory and Troy Randall's group have extended these studies to a model of allergic airway disease where we found that $\mathrm{T}$ cell-dependent lung inflammation was significantly attenuated in allergenexposed $\mathrm{Cd} 38^{--}$mice (unpublished data). Interestingly, in addition to the role that CD38 plays in regulating allergen-induced inflammation, CD38 also appears to be involved in modulating allergen-induced airway hyperresponsiveness, one of the major pathologic manifestations of asthma. In fact, prior work from the laboratories of Tim Walseth and Mathur Kannan showed that cADPR regulates calcium responses in airway smooth muscle cells (ASM) activated with a variety of spasmogens including endothelin-1 and acetylcholine (44). More recent collaborative studies between our laboratories indicated that $\mathrm{Cd} 38^{--}$mice are constitutively less responsive to contractile agonists like methacholine (45) and that this attenuated responsiveness is observed even when the ASM cells in the lungs of the $\mathrm{Cd} 38^{-/-}$mice are directly exposed to IL-13 (46). Taken together, it appears that CD38 plays multiple roles in allergic airway disease and can independently regulate the allergenmediated inflammatory response as well as the airway smooth muscle responses to contractile agonists made in response to the ongoing inflammation.

\section{CD38 Deficiency Appears to Enhance Autoimmune-mediated Tissue Damage}

While our data using $\mathrm{Cd} 38^{-/}$mice showed that $\mathrm{T}$ cell-dependent immune responses to experimental allergens were attenuated in the absence of CD38, data from several other laboratories has suggested that not all immune responses are diminished in $\mathrm{Cd} 38^{--}$mice. In fact, Teresa Martins' laboratory showed unpublished data indicating that $\mathrm{Cd} 38^{-/-}$mice expressing a mutated form of Fas ( $\mathrm{Cd} 38^{--}$mice were crossed to the autoimmune prone C57BL/6 lpr/lpr strain) not only developed a lupus-like disease, but appeared to develop disease more rapidly than the control lpr/lpr mice. Kidney dysfunction was observed in both male and female $\mathrm{Cd}_{38^{-/}}$lpr/lpr mice, suggesting the presence of autoimmune antibody complexes in the kidney. In agreement with this assessment, preliminary data revealed the deposition of immune complexes in the renal medulla of the $\mathrm{Cd} 38^{-/-} \mathrm{lpr} / \mathrm{lpr}$ animals, however, surprisingly, autoantibody production (anti-ds DNA and antiRF) was not significantly enhanced in the $\mathrm{Cd} 38^{-/-} \mathrm{lpr} / \mathrm{lpr}$ mice compared with the control lpr/lpr mice.

Although these data indicate that CD38 likely plays a protective role in pathology of this lupus-like disease, it is not immediately apparent how CD38 might mediate this protective effect. Given that CD38 is reported to be expressed on some cells in the kidney (47) and it is known that CD38/cADPR regulates calcium signaling in several non-hematopoietic cells (48, 49 ), it will be very important to determine whether the loss of CD38 on hematopoietic cells leads to an enhanced autoimmune response and accelerates the kidney damage or whether the loss of CD38 on kidney cells predisposes them to the damage mediated by the autoimmune cells and immune complexes. Indeed, in preliminary unpublished experiments from my laboratory we have found that the loss of CD38 on pancreatic 
$\beta$-islet cells increases the sensitivity of these cells to toxin-induced damage and leads to the accelerated development of diabetes, strongly suggesting that CD38 directly regulates the function of $\beta$-islet cells. In agreement with this hypothesis, recently published experiments from Jim Johnson's laboratory showed that $\beta$-cells isolated from $\mathrm{Cd} 38^{--}$mice make defective calcium responses to insulin stimulation and are more prone to apoptosis in response to several different stimuli including serum deprivation and high glucose concentrations (50). Finally, Ed Leiter and his colleagues showed that diabetes was accelerated in $\mathrm{Cd} 38^{--}$mice that were backcrossed onto the autoimmune diabetesprone NOD/Lt genetic background (51). In their experiments, they demonstrated that the loss of CD38 led to the more rapid development of diabetes and that this increased susceptibility to diabetes was dependent on expression of CD38 on the hematopoietic cells and the radiation resistant non-bone marrow derived cells such as the $\beta$-cells in the pancreas. Taken altogether, the results support the conclusion that CD38 directly regulates the survival of pancreatic $\beta$-cells exposed to toxins or autoreactive cells.

Interestingly, Leiter and colleagues also showed that the increased susceptibility of the NOD.Cd $38^{--}$mice to diabetes could be reversed when the T cell-specific mono-ADP ribosyl transferase, ART2, was deleted (51). Like CD38, ART2 utilizes $\mathrm{NAD}^{+}$as a substrate, however, unlike CD38, ART2 does not produce soluble metabolites (52). Instead, ART2

ADP-ribosylates target membrane proteins, including the purinergic receptor, P2X7 (53). Ribosylation of P2X7 by ART2expressing $\mathrm{T}$ cells leads to activation of this receptor and ultimately to $\mathrm{NAD}^{+}$induced cell death (NICD) (53). Interestingly, the loss of CD38 in the NOD mice resulted in a modest, but significant, reduction in the number of regulatory $\mathrm{T}$ cells present in these animals and this phenotype was reversed in mice that lacked both CD38 and ART2 (51). This result was perhaps not too surprising given that regulatory $\mathrm{T}$ cells are exquisitely sen- sitive to NICD (54) and that ART2-dependent NICD is enhanced in $\mathrm{Cd} 38^{-/}$mice, largely due to increased substrate availability for ART2 (55). Thus, it is reasonable to postulate that deletion of CD38 led to enhanced ART-2 dependent killing of regulatory $\mathrm{T}$ cells and resulted in a more aggressive autoimmune response. However, it is important to remember that the reduction in regulatory $\mathrm{T}$ cells seen in the $\mathrm{Cd} 38^{-/}$mice was not sufficient, in and of itself, to cause destruction of the pancreas and the loss of CD38 on nonhematopoietic radiation resistant cells, presumably the $\beta$-islet cells in the pancreas, was also necessary for the accelerated development of diabetes in this model (51). Furthermore, while the accelerated development of diabetes was very evident in the mouse model, it is not clear whether the loss or inactivation of CD38 would impact the development of type I diabetes in man as human T cells do not appear to express any of the ART family members (56). Regardless, in the future it will be important to elucidate at a molecular level how CD38 mediates its protective and damaging effects in the different inflammatory disease models.

\section{CONCLUSIONS}

Over the last fifteen years, CD38 has gone from being classified as a simple plasma membrane marker on leukocytes to being classified as a protein with a multitude of functions in cell types as diverse as pancreatic acinar cells, neurons, and leukocytes. The data presented at the 5th International CD38 meeting and the spate of recent publications focusing on CD38 indicate that the field of CD38 biology continues to grow and attract scientists from a range of disciplines. The $\mathrm{Cd}_{38^{-/}}$mouse has been a valuable tool to assess the potential in vivo roles for CD38 and to determine whether the metabolites made by CD38 regulate calcium signaling in different cell systems. Furthermore, the data from the mouse model are beginning to suggest that there is an important interplay between the ADP-ribosyl cyclase/NAD glycohydrolase family members like CD38, the mono- and poly-
ADP-ribosyl transferases, like ART2 and PARP-1, and the NAD ${ }^{+}$-dependent histone deacetylases like SIRT1 (57). In fact, recent publications from the laboratories of Jim Kirkland and Eduardo Chini indicate that $\mathrm{CD} 38$ regulates $\mathrm{NAD}^{+}$levels in a variety of tissues $(58,59)$ and that CD38 regulates the activity of SIRT1 (60). I hope that by the next meeting of CD38 biologists in 2009 we will better understand how the crosstalk between these NAD ${ }^{+}$utilizing enzymes occurs at a subcellular level and how $\mathrm{NAD}^{+}$homeostasis is regulated under normal conditions and upon inflammatory challenge. This information will likely be crucial as we try to develop drugs to manipulate the functions of these enzymes for therapeutic benefit.

\section{ACKNOWLEDGMENTS}

I would like to thank Leopoldo SantosArgumedo, Teresa Martins, Tim Walseth, Andreas Guse, Santiago Partida-Sanchez, Laura Nava-Rivero, Guixiu Shi, and Troy Randall for giving me permission to discuss their unpublished data and for reading this manuscript.

\section{REFERENCES}

1. Schuber F, Lund FE. (2004) Structure and enzymology of ADP-ribosyl cyclases: Conserved enzymes that produce multiple calcium mobilizing metabolites. Curr. Mol. Med. 4:249-61.

2. Liu $Q$ et al. (2005) Crystal structure of human CD38 extracellular domain. Structure (Camb.) 13:1331-9.

3. Lee HC, Walseth TF, Bratt GT, Hayes RN, Clapper DL. (1989) Structural determination of a cyclic metabolite of $\mathrm{NAD}^{+}$with intracellular $\mathrm{Ca}^{2+}$ mobilizing activity. J. Biol. Chem. 264:1608-15.

4. Lee HC. (2001) Physiological functions of cyclic ADP-ribose and NAADP as calcium messengers. Annu. Rev. Pharmacol. Toxicol. 41:317-45.

5. Walseth TF, Lee HC. (2002) Pharmacology of Cyclic ADP-ribose and NAADP: Synthesis and properties of analogues. In: Lee HC (ed.) Cyclic ADP-ribose and NAADP. Structures, Metabolism and Functions. Kluwer Academic Publishers, Boston MA, pp. 121-42.

6. Potter BV, Walseth TF. (2004) Medicinal chemistry and pharmacology of cyclic ADP-ribose. Curr. Mol. Med. 4:303-11.

7. Guse AH. (2005) Second messenger function and the structure-activity relationship of cyclic adenosine diphosphoribose (cADPR). Febs. J. 272:4590-7.

8. Lee HC, Aarhus R. (1995) A derivative of NADP mobilizes calcium stores insensitive to inositol trisphosphate and cyclic ADP-ribose. J. Biol. Chem. 270:2152-7.

9. Howard M et al. (1993) Formation and hydroly- 
sis of cyclic ADP-ribose catalyzed by lymphocyte antigen CD38. Science 262:1056-9.

10. Deaglio S, Vaisitti T, Aydin S, Ferrero E, Malavasi F. (2006) In-tandem insight from basic science combined with clinical research: CD38 as both marker and key component of the pathogenetic network underlying chronic lymphocytic leukemia. Blood 108:1135-44.

11. Santos-Argumedo L, Teixeira C, Preece G, Kirkham PA, Parkhouse RME. (1993) A B lymphocyte surface molecule mediating activation and protection from apoptosis via calcium channels. J. Immunol. 151:3119-30.

12. Harada $\mathrm{N}$ et al. (1993) Expression cloning of a cDNA encoding a novel murine B cell activation marker. J. Immunol. 151:3111-8.

13. Lund FE et al. (1996) Signaling through CD38 augments B cell antigen receptor (BCR) responses and is dependent on BCR expression. J. Immunol. 157:1455-67.

14. Moreno-Garcia ME et al. (2005) CD38 signaling regulates $\mathrm{B}$ lymphocyte activation via a phospholipase C (PLC)-gamma 2-independent, protein kinase $C$, phosphatidylcholine-PLC, and phospholipase D-dependent signaling cascade. J. Immunol. 174:2687-95.

15. Hoshino K et al. (1999) Cutting edge: Toll-like receptor 4 (TLR4)-deficient mice are hyporesponsive to lipopolysaccharide: evidence for TLR4 as the Lps gene product. J. Immunol. 162:3749-52.

16. Krieg AM et al. (1995) CpG motifs in bacterial DNA trigger direct B-cell activation. Nature 374:546-9.

17. Alexopoulou L, Holt AC, Medzhitov R, Flavell RA. (2001) Recognition of double-stranded RNA and activation of NF-kappaB by Toll-like receptor 3. Nature 413:732-8.

18. Lund JM et al. (2004) Recognition of singlestranded RNA viruses by Toll-like receptor 7 . Proc. Natl. Acad. Sci. USA 101: 5598-603.

19. Yasue $\mathrm{T}$ et al. (1999) $\operatorname{IgG} 1$ production by $\operatorname{sg} \mathrm{D}^{+}$ splenic B cells and peritoneal B-1 cells in response to IL-5 and CD38 ligation. Int. Immunol. 11:915-23.

20. Santos-Argumedo L et al. (1994) CD38 unresponsiveness of xid B cells implicates Bruton's tyrosine kinase $(b t k)$ as a regulator of CD38 induced signal transduction. Int. Immunol. 7:163-70.

21. Lund FE et al. (2006) CD38 induces apoptosis of a murine pro-B leukemic cell line by a tyrosine kinase-dependent but ADP-ribosyl cyclase- and NAD glycohydrolase-independent mechanism. Int. Immunol. 18:1029-42.

22. Muller-Steffner H, Malver O, Oppenheimer NJ, Schuber F. (1992) Slow-binding inhibition of $\mathrm{NAD}^{+}$glycohydrolase by arabino analogues of $\beta$-NAD ${ }^{+}$. J. Biol. Chem. 267:9606-11.

23. Berthelier V, Tixier J-M, Muller-Steffner H, Schuber F, Deterre P. (1998) Human CD38 is an authentic NAD $(\mathrm{P})^{+}$glycohydrolase. Biochem. J. 330:1383-90.

24. Zubiaur M et al. (2002) CD38 is associated with lipid rafts and upon receptor stimulation leads to Akt/Protein Kinase B and erk activation in the absence of the CD3- $\zeta$ immune receptor tyrosinebased activation motifs. J. Biol. Chem. 277:13-22.

25. Munoz P et al. (2003) CD38 signaling in T cells is initiated within a subset of membrane rafts con- taining Lck and the CD3-zeta subunit of the T cell antigen receptor. J. Biol. Chem. 278:50791-802.

26. Pavon EJ et al. (2006) Increased association of CD38 with lipid rafts in T cells from patients with systemic lupus erythematosus and in activated normal T cells. Mol. Immunol. 43:1029-39.

27. Lund FE et al. (1998) CD38: A new paradigm in lymphocyte activation and signal transduction. Immunol. Rev. 161:79-93.

28. Partida-Sanchez S, Randall TD, Lund FE. (2003) Innate immunity is regulated by CD38, an ectoenzyme with ADP-ribosyl cyclase activity. $\mathrm{Mi}$ crobes Infect. 5:49-58.

29. Hopken UE, Lipp M. (2004) All roads lead to Rome: triggering dendritic cell migration. Immunity 20:244-6.

30. Partida-Sanchez S et al. (2001) Cyclic ADP-ribose production by CD38 regulates intracellular calcium release, extracellular calcium influx and chemotaxis in neutrophils and is required for bacterial clearance in vivo. Nat. Med. 7:1209-16.

31. Partida-Sanchez S et al. (2004) Regulation of dendritic cell trafficking by the ADP-ribosyl cyclase CD38: Impact on the development of humoral immunity. Immunity 20:279-91.

32. Partida-Sanchez S et al. (2004) Chemotaxis and calcium responses of phagocytes to formyl-peptide receptor ligands is differentially regulated by cyclic ADP-ribose. J. Immunol. 172:1896-1906.

33. Clapper DL, Walseth TF, Dargie PJ, Lee HC. (1987) Pyridine nucleotide metabolites stimulate calcium release from sea urchin egg microsomes desensitized to inositol trisphosphate. J. Biol. Chem. 262:9561-8.

34. Guse AH et al. (1999) Regulation of calcium signaling in T lymphocytes by the second messenger cyclic ADP-ribose. Nature 398:70-3.

35. Sano $\mathrm{Y}$ et al. (2001) Immunocyte $\mathrm{Ca}^{2+}$ influx system mediated by LTRPC2. Science 293:1327-30.

36. Perraud AL et al. (2001) ADP-ribose gating of the calcium-permeable LTRPC2 channel revealed by Nudix motif homology. Nature 411:595-9.

37. Hara $\mathrm{Y}$ et al. (2002) LTRPC2 $\mathrm{Ca}^{2+}$-permeable channel activated by changes in redox status confers susceptibility to cell death. Mol. Cell. 9:163-73.

38. Kolisek M, Beck A, Fleig A, Penner R. (2005) Cyclic ADP-ribose and hydrogen peroxide synergize with ADP-ribose in the activation of TRPM2 channels. Mol. Cell. 18:61-9.

39. Shen BW, Perraud AL, Scharenberg A, Stoddard BL. (2003) The crystal structure and mutational analysis of human NUDT9. J. Mol. Biol. 332:385-98.

40. McHugh D, Flemming R, Xu SZ, Perraud AL, Beech DJ. (2003) Critical intracellular Ca2+ dependence of transient receptor potential melastatin 2 (TRPM2) cation channel activation. J. Biol. Chem. 278: 11002-6.

41. Togashi K et al. (2006) TRPM2 activation by cyclic ADP-ribose at body temperature is involved in insulin secretion. EMBO J. 25: 1804-15.

42. Beck A, Kolisek M, Bagley LA, Fleig A, Penner R (2006) Nicotinic acid adenine dinucleotide phosphate and cyclic ADP-ribose regulate TRPM2 channels in T lymphocytes. FASEB J. 20: 962-4.

43. Cockayne D et al. (1998) Mice deficient for the
ecto-NAD ${ }^{+}$glycohydrolase CD38 exhibit altered humoral immune responses. Blood 92:1324-33.

44. Deshpande DA et al. (2005) CD38/cyclic ADP-ribose signaling: role in the regulation of calcium homeostasis in airway smooth muscle. Am. J. Physiol. Lung Cell. Mol. Physiol. 288:L773-88.

45. Deshpande DA et al. (2004) Altered airway responsiveness in CD38-deficient mice. Am. J. Respir. Cell Mol. Biol. 32:149-56.

46. Guedes AG et al. (2006) CD38-deficient mice have reduced airway hyperresponsiveness following IL-13 challenge. Am. J. Physiol. Lung Cell. Mol. Physiol. 241:1286-93

47. Fernandez JE et al. (1998) Analysis of the distribution of human CD38 and of its ligand CD31 in normal tissues. J. Biol. Reg. Homeost. Agents 12:81-91.

48. Guse AH. (2004) Regulation of calcium signaling by the second messenger cyclic adenosine diphosphoribose (cADPR). Curr. Mol. Med. 4:239-48.

49. Kato I et al. (1999) CD38 disruption impairs glucose-induced increases in cyclic ADP-ribose, $\left[\mathrm{Ca}^{2+}\right]_{i^{\prime}}$ and insulin secretion. J. Biol. Chem. 274:1869-72.

50. Johnson JD et al. (2006) Suppressed insulin signaling and increased apoptosis in Cd38-null islets. Diabetes 55:2737-46.

51. Chen J et al. (2006) Targeted disruption of CD38 accelerates autoimmune diabetes in NOD/Lt mice by enhancing autoimmunity in an ADPribosyltransferase 2-dependent fashion. J. Immunol. 176:4590-9.

52. Koch-Nolte F et al. (2006) ADP-ribosylation of membrane proteins: unveiling the secrets of a crucial regulatory mechanism in mammalian cells. Ann. Med. 38:188-99.

53. Seman $\mathrm{M}$ et al. (2003) NAD-induced T cell death: ADP-ribosylation of cell surface proteins by ART2 activates the cytolytic P2X7 purinoceptor. Immunity 19:571-82.

54. Aswad F, Kawamura H, Dennert G. (2005) High sensitivity of CD4+CD25+ regulatory T cells to extracellular metabolites nicotinamide adenine dinucleotide and ATP: a role for P2X7 receptors. J. Immunol. 175:3075-83.

55. Krebs C et al. (2005) CD38 controls ADPribosyltransferase-2-catalyzed ADP-ribosylation of T cell surface proteins. J. Immunol. 174:3298-305.

56. Seman M, Adriouch S, Haag F, Koch-Nolte F (2004) Ecto-ADP-ribosyltransferases (ARTs): emerging actors in cell communication and signaling. Curr. Med. Chem. 11:857-72.

57. Bordone L, Guarente L. (2005) Calorie restriction, SIRT1 and metabolism: understanding longevity. Nat. Rev. Mol. Cell. Biol. 6:298-305.

58. Young GS, Choleris E, Lund FE, Kirkland JB. (2006) Decreased cADPR and increased NAD+ in the Cd38-/- mouse. Biochem. Biophys. Res. Commun. 346:188-92.

59. Aksoy P, White TA, Thompson M, Chini EN. (2006) Regulation of intracellular levels of NAD: a novel role for CD38. Biochem. Biophys. Res. Commun. 345:1386-92.

60. Aksoy P et al. (2006) Regulation of SIRT 1 mediated NAD dependent deacetylation: A novel role for the multifunctional enzyme CD38. Biochem. Biophys. Res. Commun. 349:353-9. 